\title{
To Detection of Fish Disease using Augmented Reality and Image Processing
}

\author{
Hitesh Chakravorty \\ hitesh_chtvy@yahoo.co.in
}

\begin{abstract}
Fish mortality due to diseases. The diseased identification at early stage to prevent and spreading of diseases. The image processing technique is already existed to detect fish disease. The Augmented model and Image Processing a new approach to detect fish disease using smart phone.The comparison of the disease fish on the spot is not possible in image processing technique. The AR model technique has over come on the spot identification of disease fish. Taken one Epizootic Ulcerative Syndrome (EUS) diseased fish model for the proposed approach. The images taken with the AR model for image processin HSV operation for better accuracy to diseased area detection.
\end{abstract}

Key words- Augmented Reality(AR),Blender, Unity, Vuforia, Python

\section{Introduction}

The fresh water fish dies unnaturally due to disease cause by Epizootic Ulcerative Syndrome (EUS) has been increased since 1988. The red mark appear on the surface of the fish, leads to fall off scale expose the body. Later develop ulcer fish dies within few days. The early detection can prevent fish mortality as well as increase production. To identify disease fish with necked eye are not error free. To over come these lacuna new technology such as Image Processing Technique, Machine Learning come into existence [1] [2].

The Augmented Reality comes into existence just a few decade ago. Its application has growing rapidly in the recent years. Augmented Reality generates a live vision of the real-world with virtual objects or information of physical objects or environments, resulting in a mixed reality. To applying this concept numerous mobile application come into such as navigation, advertisement, display information, education, creativity and games [3] [4].

In this paper a new and combine technique of Augmented Reality and Image Processing is adopted to identify Epizootic Ulcerative Syndrome (EUS) of Puntius chola.

\section{Materials and Methods}

Fish effected with Epizootic Ulcerative Syndrome (EUS) of Puntius chola was collected from Hailakandi, Assam and identified by human expert.Using disease fish image to make fish 3Dmodel for AR Model App. Pictures(FIGURE1) of fish were taken by the AR Mobile App. Images were 200 by 200 pixels so that an engineering compromise can be obtained between processing time of algorithms and clarity retention of input images. 

in Image and Video Processing, Volume 7 No 6, Dec (2019); pp: 1-4

In this project needs the following capabilities software requirements - Blender2.8,Unity3D, Operating System- Windows 1064 bit. Hardware requirements includes 7th Generation Intel core processor, 4 GB RAM, 8GB memory, Mobile capable run Vuforia AR App.

To conduct Image Processing using OpenCV and Python.

\subsection{Feature Extraction Using HSV}

The HSV space component to reduce computation and improve efficiency. In accordance with the different colors and subjective color perception quantification, quantified hue $(H)$, saturation $(S)$ and intensity (V) are obtained. Hue ranges from 0 to 360 degrees, with variation beginning with red, going through yellow, green, cyan, blue and magenta and black to red[5].

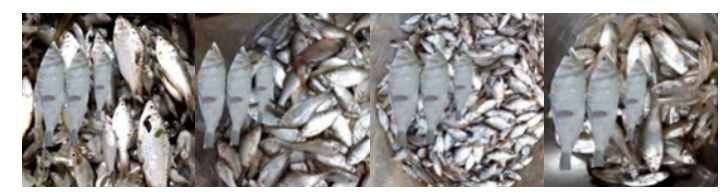

Figure 1: Sample Image Taken with AR App

\section{Experimental Finding}

In order to address the hypothesis that the detect disease fish by user using AR model (FIGURE2)

1) disease fish 3D model made using Blender 2.8

2) creating App for smart phone using Unity and Vuforia to display an Augmented model

3) image taken to compare fish with Augmented model visually and for Image Processing purpose

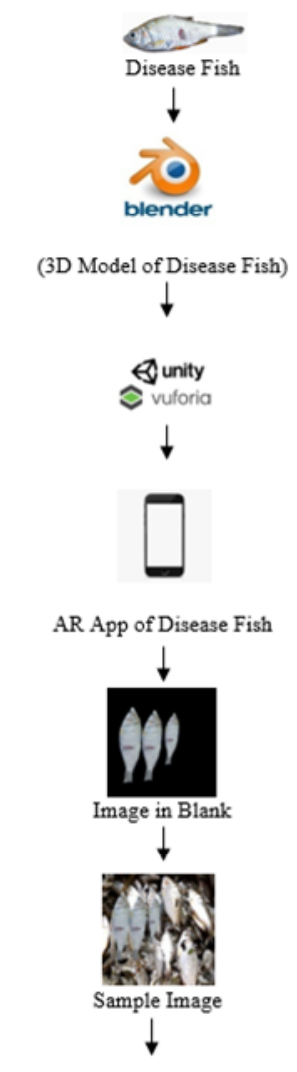

Figure2: Flow chart for disease fish to comparison with sample fishes Image Processing of Sample Image 


\subsection{HSV and Segmentation}

For HSV, Hue range is $[0,179]$, Saturation range is $[0,255]$ and Value range is $[0,255]$. Different softwares use different scales. To convert BGR image to HSV, can use to extract a colored object. In HSV, it is more easier to represent a color than RGB color-space. In this application extract a blue colored of the effected fish. The method used here (FIGURE3)

1) each sample image convert from BGR to HSV color-space,

2) the threshold HSV image for a range of blue color,

3) extract the blue region represent the disease effected area

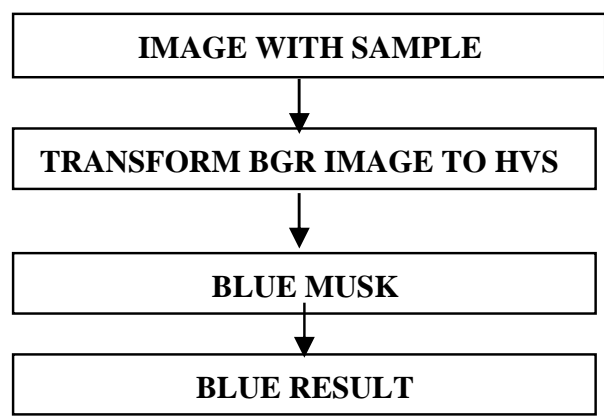

Figure 3: Flow chart for experimentation HSV and blue region Complete Process of HSV
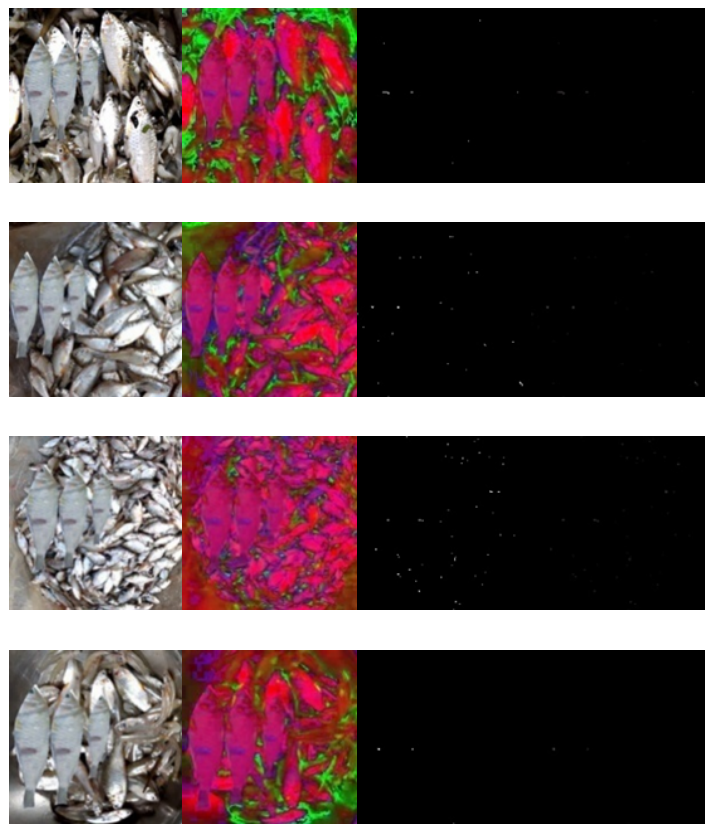

(a)

(b)

(c)

(d)

Figure 4: (a)Image Sample (b) HVS of Sample (c) Blue Musk (d)Blue Result

\section{Results and Discussion}

The image processing technique already exist to fish disease identification, is not perfect and ideal method.The present approach step forward to improvement of fish disease identification and prevention.

The three disease fish 3Dmodel of different size use in AR applications of Puntius chola for visuallay comparing detecting disease fish on the spot.To compare AR App with sample fish no Puntius chola effected with Epizootic Ulcerative Syndrome (EUS).

HSV diseased fish images show the diseased area clearly in AR model. HSV indicates the dominant color of fish diseased area. The experimental results suggest that the introduced method for diseased 

in Image and Video Processing, Volume 7 No 6, Dec (2019); pp: 1-4

segmentation accurately segment the diseased portion. In the sample fish no disease fish is found (FIGURE4).

\section{Conclusion}

The identifying fish diseases is major concern and improvement of fish production. The approach made in this paper make a new dimension to detect fish disease. The one disease fish of Augmented model and Image Processing for this present experiment. The result show that no disease fish.

This experiment for limited mobile devices with hardware capabilities. The three disease fish 3D model of different size use in AR applications it can be made improved using perfect disease fish 3D model.

In future overcome the limitation the Augmented Reality and Image Processing technique has brighter scope for fish disease identification.

\section{REFERENCES}

[1] D. Kar, S.C.Dey and A. Roy, 2000. "Epizootic Ulcerative Syndrome In Fish at Barak Valley, Assam, India", Section-8 Sustainable Water Resource Management, Policies And Protocols.

[2] H. Chakravorty, R. Paul and P. Das, 2015, "Image Processing Technique to Detect Fish Disease",International Journal of Computer Science and Security (IJCSS), Volume (9) : Issue (2) : 2015, pp 121-131.

[3] Chen P., Liu X., Cheng W., Huang R. (2017) "A review of using Augmented Reality in Education from 2011 to 2016". School of Educational Technology, Faculty of Education, Beijing Normal University,Beijing, China, Smart Learning Institute, Beijing Normal University, Beijing, China, Departments of Educational Technology, Capital Normal University, Beijing, China In: Popescu E. et al. (eds) Innovations in Smart Learning. Lecture Notes in Educational Technology. Springer, Singapore

[4] Luke Benjamin Cassar and Frankie Inguanez (2018) "ARC: Augmented Reality for Catering",Institute of Information \& Communication Technology University College Malta College of Arts, Science \& Technology, Paola PLA9032, Malta, 2018 IEEE 8th International Conference on Consumer Electronics - Berlin (ICCE-Berlin). 978-1-5386-6095-9/18/\$31.00 C2018 IEEE

[5] https://opencv-python-

tutroals.readthedocs.io/en/latest/py_tutorials/py_imgproc/py_colorspaces/py_colorspaces.html 